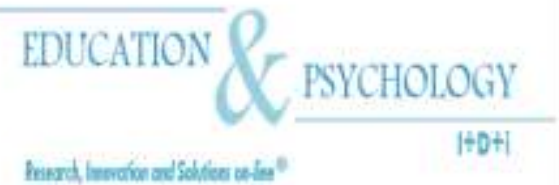

\title{
La contribución del Edublog como estrategia didáctica
}

\section{José Francisco Durán Medina} Departamento de Pedagogía, Universidad de Castilla La Mancha,
Toledo

\section{España}

Correspondencia: José Francisco Durán Medina. C/ Príncipe, 26. 45400 Mora (Toledo). España. E-mail: JoseFrancisco.Duran@uclm.es

(C) Education \& Psychology I+D+i and Editorial EOS (Spain) 


\section{Resumen}

Introducción. Este Trabajo de Investigación forma parte de la tesis que lleva por título "Los edublogs como agentes potenciadores de la motivación y favorecedores de las relaciones personales en los procesos de Enseñanza-Aprendizaje", realizada por el autor del artículo, cuyo objetivo general no es otro sino contribuir a una posible mejora de la calidad en la enseñanza.

Método. En este caso se ha optado por dotar al cuestionario utilizado con la mayor rigurosidad, fiabilidad y validez posibles mediante un método de expertos, el método Delphi, utilizando como fuente de información un grupo de personas a las que se supone un conocimiento elevado de la materia que se va a tratar.

Resultados. Para realizar el análisis descriptivo de datos se han utilizado tablas de frecuencia con gráficos, medidas de tendencia central, histogramas, variabilidad, asimetría y curtosis. De igual manera, se han llevado a cabo diversas pruebas que han ayudado en la investigación del tema que nos ocupa. Por un lado, se establecieron relaciones de contenidos entre sí intentando descubrir la existencia o no de dicha dependencia. Posteriormente, se averiguaron los coeficientes de correlación mediante modelos de regresión tanto paramétricos como no paramétricos, pudiendo utilizarse los resultados obtenidos para las posibles predicciones.

Discusión y conclusiones La utilización del edublog, independientemente de otras muchas variables (como pueden ser el sexo, la obligatoriedad de los estudios, tipo de localidad, nivel de utilización, etc.), sí resulta eficaz como estrategia didáctica facilitadora de adquisición de contenidos.

Palabras Clave: Blog, Edublog, Estrategia Didáctica, Adquisición de Contenidos, Pedagogía.

Recibido: 06/08/10 Aceptación Inicial: 22/08/10 Aceptación Definitiva: 17/11/10 


\title{
The contribution of the Edublog as a teaching strategy
}

\begin{abstract}
Introduction. This Work of Investigation comprises of the thesis titled "Edublogs like harnesser agents of the motivation and enhancing of the personal relations in the processes of Education-Learning", realised by the author of the article, whose general mission is not another one but to contribute to a possible improvement of the quality in education.
\end{abstract}

Method. In this case it has been chosen to equip to the questionnaire used with the greater rigor, possible reliability and validity by means of a method of experts, the Delphi method, using like source of intelligence a group of people to whom a lifted knowledge of the matter assumes that is going away to try.

Results. In order to realise the descriptive analysis of data, tables of frequency with graphs has been used, measures of central tendency, histograms, variability, asymmetry and curtosis. Of equal way, diverse tests have been carried out that have helped in the investigation of the subject that occupies to us. On the one hand, relations of contents settled down to each other trying to discover the existence or not of this dependency. Later, the coefficients of correlation were found out by means of nonparametric and parametric models of regression, being able to be used the results obtained for the possible predictions.

Discussion or Conclusion. The use of edublog, independent of other many variables (as they can be sex, the obligatory nature of the studies, type of locality, level of use, etc.), is effective like facilitater didactic strategy of acquisition of contents.

Keywords: Blog, Edublog, Didactic Strategy, Acquisition of Contents, Pedagogy.

Received: 08/06/10 Initial Acceptance: 08/22/10 Definitive Acceptance: 11/17/10 


\section{Introducción}

Este Trabajo de Investigación forma parte de la tesis que lleva por título "Los edublogs como agentes potenciadores de la motivación y favorecedores de las relaciones personales en los procesos de Enseñanza-Aprendizaje, cuyo objetivo general no es otro sino contribuir a una posible mejora de la calidad en la enseñanza.

Todos los docentes debemos hacer lo posible para que la mayoría del alumnado alcance los objetivos propuestos o, al menos, lo intente. No es suficiente con las numerosas excusas de culpar a la sociedad, a la escasa presencia en el hogar de los padres y madres por motivos laborales, al exceso de violencia en la televisión y videojuegos... Basta ya de echar balones a otros tejados, ya está bien de ver la paja en el ojo ajeno y no ver la viga en los propios. Si bien es verdad que todo esto merma en gran cantidad la calidad del proceso educativo, no es menos cierto que como docentes debemos procurar eliminar sus efectos negativos, acopiarnos de valor y energía, y probar otros métodos, estrategias nuevas, recursos atrayentes y motivadores como los edublogs u otras TIC (Tecnologías de la Información y Comunicación), para conseguir con ello lo que verdaderamente pretendemos: dar rienda suelta a nuestra vocación y... enseñar; enseñar a ser personas, a respetar a los demás, a saber convivir, a ser solidarios, a no transgredir la libertad de otros. En definitiva, educar.

Ni qué decir tiene los beneficios que esto aportaría a la sociedad, si bien no queremos pecar de utópicos, dejando constancia de que la única pretensión que se persigue es conseguir una pequeña mejora, no un imposible cambio radical instantáneo. Si las conclusiones son propicias y los resultados alentadores, entonces poco a poco, ladrillo a ladrillo, podremos ir construyendo entre todos ese centro educativo que anhelamos, esa sociedad respetuosa y solidaria que deseamos. Cuanto menos, tendremos la esperanza y seguridad de que es posible.

Muchos autores de reconocido prestigio apoyan la incorporación y utilización de edublogs y otras TICs en las aulas previendo las modificaciones que tendrá que efectuar la educación haciendo uso de éstas para poder seguir en lo posible los rápidos cambios que ya se están dando en la sociedad digital. Entre tantos que se podrían citar (Pere Marqués, Manuel Área Moreira, Julio Cabero, etc.) detallamos a continuación algunos de gran interés para nuestro estudio: 
Gértrudix y Gértrudix (2007) hacen hincapié en las transformaciones tecnológicas y sociales que se están produciendo en los inicios del siglo XXI, afectando sin duda a la educación de múltiples formas. Opinan que este desarrollo tecnológico afecta ya a todas las áreas de la vida y a todos los miembros de la comunidad, y en el futuro inmediato es previsible que lo haga aún más. El alumnado, como ciudadanos del siglo XXI, tiene unas necesidades, unas motivaciones y una actitud hacia las TIC completamente distintas que el de hace tan sólo unos años. El aumento de las dotaciones que están llevando a cabo los gobiernos, el valor que cada vez más se otorga al uso didáctico de éstas, y la capital importancia que han acabado por poseer en todas las esferas (social, económica, ocio, comunicación...) ponen el acento en la necesidad de conocer cuáles son las formas más eficientes de utilización para favorecer los procesos de Enseñanza-Aprendizaje. En este sentido, la mayor parte de los estudios realizados, tanto desde el ámbito académico como desde las instancias administrativas, ofrecen ya un catálogo de recomendaciones de interés en relación a los despliegues y al fomento de su uso y aprovechamiento.

Del mismo modo, Gómez (2004) comenta que en este nuevo contexto donde aparecen con fuerza las TIC, la pregunta pasa a ser cuál es el valor añadido que aportan estas nuevas tecnologías (correo electrónico, grupos de noticias, fórums, web, incluso podríamos añadir los blogs). Su respuesta: potencian la interactividad, y en consecuencia el desarrollo sociocognitivo de alumnos y una actitud más positiva hacia las matemáticas, atreviéndonos a añadir que de la misma manera, hacia otras asignaturas.

También García y Romero (2009), coincidiendo con los resultados de este estudio, constatan en una de sus investigaciones la influencia de las TIC en las actitudes de los alumnos, en este caso, de secundaria.

González (2002), incide en la variedad de modalidades de comunicación que utiliza el lenguaje multimedia (visual, auditiva, gráfica y recursos telemáticos), convirtiéndolo en un buen aliado para promover la comunicación didáctica con los destinatarios de los programas educativos, lúdicos, sociales y culturales de los centros educativos. Nos señala que este nuevo lenguaje crea un contexto motivador y ofrece posibilidades para su integración y utilización tanto para propósitos individuales como en la dinámica grupal que se planifique por parte de los técnicos, formadores y monitores. 
Sánchez (1999) comparte la misma idea sobre la necesidad de incorporar las TIC en las aulas, manifestando la principal ventaja que las NNTT (nuevas tecnologías) pueden aportar a la educación: el incremento considerable de la información que se pone a disposición de los profesores y sus alumnos. Esta información puede ser recibida en muy diversos códigos y, en algunas ocasiones, ser el único medio para poder ser recibidas. La información ya no se localiza en un lugar determinado, lo que lleva a la ruptura de barreras espacio-temporales y a un nuevo modo de construir el conocimiento, favoreciendo el trabajo colaborativo y el autoaprendizaje de los alumnos.

Centrándonos concretamente en la utilización de edublogs en centros educativos podemos exponer ciertas experiencias internacionales que demuestran su integración en este campo con importantes y óptimos resultados:

Michel (2009), ha diseñado un blog que se convierte en propuesta interactiva de la reforma educativa en Educación Primaria de México. Uribe (2009) también expuso con claridad durante el IV Congreso de la Cibersociedad 2009 el impacto de los edublogs en los procesos educativos, relacionándolos con la inteligencia colectiva. Del mismo modo, García (2008) realiza un estudio sobre los edublogs como enfoque globalizador de las competencias básicas en el currículo de lengua extranjera, conectando el tema con la actualidad reciente donde imperan las competencias en cualquier programación didáctica escolar.

En otra línea, igualmente destacable, existen edublogs con la intención de potenciar los importantes lazos de unión y coordinación entre padres, madres, apoderados, apoderadas y los profesores. Un ejemplo de Chile: http://www.convivenciaescolar.cl/Blog_de_Blogs/24Blog_Padres_Madres_Apoderados_y_Apoderadas/. Para encontrar una relación de artículos interesantes sobre los edublogs y sus aplicaciones en los centros educativos se recomienda visitar http://dialnet.unirioja.es/servlet/busquedadoc? $\mathrm{db}=1 \& \mathrm{t}=$ edublogs \&td=todo.

Siguiendo la misma línea de éstos y otros muchos autores de idéntica opinión, la pretensión de este trabajo de investigación sobre la influencia de los edublogs en los procesos de Enseñanza-Aprendizaje, sin ánimo de parecer utópica, sería intentar acrecentar los lazos de unión entre profesor y alumno, modernizando y acercando el proceso de enseñanzaaprendizaje a la realidad actual y entorno más próximo de los discentes, procurando conseguir 
la atención de los mismos mediante el uso de recursos atractivos habitualmente utilizados por ellos.

Verdaderamente no es tarea fácil, pero seguramente resultará gratificante y muy prometedor cualquier avance en este sentido por pequeño que sea, con la esperanza de averiguar si la utilización en entornos educativos de los blogs pudiera conseguir ese ansiado aumento de motivación, aportando cercanía en las relaciones personales y contribuyendo a una apreciable mejoría en el rendimiento académico de los alumnos.

Sería deseable averiguar si la utilización de un edublog puede servir como estrategia didáctica facilitadora de adquisición de contenidos facilitando la puesta en práctica de dichos contenidos, facilitando también la integración y aprendizaje de contenidos transversales, ayudando al desarrollo de la habilidad lectora y escritora, así como del desarrollo de la expresión oral, favoreciendo la creación de Comunidades de estudiantes y la construcción de nuevos conocimientos (nuevo pensamiento).

\section{Objetivos}

El objetivo principal de este trabajo de investigación es la búsqueda de respuestas a las preguntas de investigación realizadas, planteándonos si la utilización de un edublog en el aula... ¿favorece la adquisición de contenidos de la asignatura?, ¿facilita la puesta en práctica de contenidos de la asignatura?, ¿facilita la integración y aprendizaje de contenidos transversales?, ¿ayuda al desarrollo de la habilidad lectora?, ¿ayuda al desarrollo de la habilidad escritora?, ¿ayuda al desarrollo de la expresión oral?, ¿favorece la creación de Comunidades de estudiantes?, ¿favorece la construcción de nuevos conocimientos (nuevo pensamiento)?

Por tanto, nuestro objetivo general podría desgranarse en ocho objetivos más específicos o concretos, valorables mediante la opinión del alumnado y profesorado participante en la investigación sobre las ventajas que aportan la utilización de edublogs en las aulas:

1. Comprobar si la utilización de un edublog en el aula favorece la adquisición de contenidos de la asignatura. 
2. Comprobar si la utilización de un edublog en el aula facilita la puesta en práctica de contenidos de la asignatura.

3. Comprobar si la utilización de un edublog en el aula facilita la integración y aprendizaje de contenidos transversales.

4. Comprobar si la utilización de un edublog en el aula ayuda al desarrollo de la habilidad lectora.

5. Comprobar si la utilización de un edublog en el aula ayuda al desarrollo de la habilidad escritora.

6. Comprobar si la utilización de un edublog en el aula ayuda al desarrollo de la expresión oral.

7. Comprobar si la utilización de un edublog en el aula favorece la creación de Comunidades de estudiantes.

8. Comprobar si la utilización de un edublog en el aula favorece la construcción de nuevos conocimientos (nuevo pensamiento).

\section{Método}

\section{Participantes}

En cuanto al muestreo, distinguiremos cinco elementos en su proceso: el universo, la población, la muestra invitada, la muestra aceptante y la muestra productora de datos. Nuestro heterogéneo universo podríamos subdividirlo en varios universos a su vez: universo objetivo, perteneciendo al mismo todos los alumnos a nivel mundial que participan en un edublog de aula; y universo muestral, con todos los alumnos de los países colaboradores que participan en un edublog de aula.

La población, es decir, la parte del universo a la que el investigador tiene acceso, la compondrían los alumnos que participan en un edublog de aula actual y activo, localizable a 
través de Internet. La muestra de esta investigación fue tomada de manera aleatoria entre los distintos edublogs accesibles por Internet a través de buscadores, listas de distribución, edublogs escolares de varias páginas educativas...

En la muestra invitada se encuentran los alumnos de los profesores autores de los edublogs que se han prestado voluntariamente a colaborar en la investigación, hayan aceptado o no la inclusión en la misma: 2559 usuarios. La muestra aceptante está compuesta por los alumnos de los profesores autores de los edublogs que aceptaron participar en la investigación, rellenaran o no, correcta o incorrectamente (no válidos), los cuestionarios.Por último, la muestra productora de datos (coincidente en este caso con la muestra aceptante) son todos los alumnos que han participado y colaborado realmente en la investigación, es decir, 1161 alumnos.

Un total de tres continentes distintos (Europa, América del Norte y del Sur, Oceanía), ocho países diferentes (Alemania y España; México, Argentina, Chile, Colombia y Puerto Rico; Australia), 23 comunidades o distritos, 65 localidades, 74 centros educativos, 1161 alumnos que han rellenado los cuestionarios. Éstos son los datos numéricos globales de los usuarios de edublog participantes en la investigación.

\section{Instrumentos}

Las limitaciones de los modelos de procesamiento de la información para aportar conocimientos útiles desde el punto de vista de la formación y el desarrollo profesional del docente ha dado lugar a la aparición de una serie de líneas de trabajo que abogan por la necesidad de analizar y comprender las vivencias, percepciones, sentimientos y conocimientos que docentes y alumnos activan cuando se enfrentan a las situaciones de su trabajo cotidiano (Clandinin y Conelly, 1986).

Para ello se han utilizado diversas técnicas en multitud de investigaciones: cuestionarios, parrilla de Kelly, entrevista, observación, estudio de casos, análisis de documentos, grupos de discusión, inventarios, árboles ordenados, estudio longitudinal, seminarios, grabación en vídeo. 
Entrevistas y cuestionarios han sido muy utilizados en diversas e importantes investigaciones tanrto nacionales como internacionales, como por ejemplo, la realizada por Dilmac (2009) utilizando una encuesta como método de relación de la investigación cuantitativa respecto a las tendencias generales de los profesores, realizando un exhaustivo análisis de los mismos.

Nosotros vamos a centrar nuestra atención en los cuestionarios, instrumento con el que se han recogido los datos de esta investigación. El cuestionario puede considerarse como una forma de entrevista por escrito.

El procedimiento para la elaboración de un cuestionario es de primordial importancia para solventar cualquier problema y evitar errores insalvables posteriormente.

Los cuestionarios fueron contestados por los propios alumnos usuarios de los edublogs de una manera anónima para garantizar su veracidad, y dejando espacios para que puedan aportar algunas observaciones si lo creen oportuno. Debemos destacar que, al tratarse de una franja de edades bastante amplia, abarcando desde los niveles de primaria hasta los universitarios, y dadas las grandes diferencias existentes entre ellos, sobre todo en cuanto a la comprensión de textos escritos y amplitud de vocabulario se refiere, se han utilizado dos cuestionarios: uno para los niveles de Enseñanza Obligatoria, y otro para los de Enseñanza no Obligatoria. Incluso, se ha utilizado en alguna ocasión uno de ellos traducido al inglés para su mejor comprensión internacional. Aunque las únicas diferencias entre ambos cuestionarios se basaban en los ítems acerca del nivel de estudios, sí hay que destacar la conveniencia de precisar y explicar algunas de las preguntas a los alumnos de más corta edad para su mejor comprensión.

Se considera importante señalar que únicamente se ofrecieron cuatro respuestas posibles: totalmente de acuerdo, medianamente de acuerdo, medianamente en desacuerdo, totalmente en desacuerdo. La reducción de las respuestas a un número de cuatro se realizó con la intención de no posibilitar una respuesta intermedia imprecisa, obligando de esta manera a las muestras a una inclinación hacia una u otra opción, a favor o en contra, haciendo los resultados más categóricos.

El cuestionario resultante y utilizado para toda la investigación se encuentra en el anexo I, al final de este trabajo. Los resultados fueron sometidos a las pertinentes pruebas (coeficiente de correlación de Pearson, coeficiente de correlación lineal rho de Spearman, 
coeficiente de correlación tau de kendall, prueba de Chi-cuadrado) arrojando unos resultados notables dejando patente tanto su validez como su fiabilidad. Del mismo modo que se hace con los cuestionarios, alguna muestra de estas pruebas pueden observarse en los anexos II y III (Correlaciones y Chi-cuadrado) al final del trabajo, constatando la inexistencia de correlación positiva entre las variables (edad, sexo, estudios...) y el aspecto educativo estudiado: la adquisición de contenidos, incidiendo así en la validez de los resultados obtenidos y comparando los resultados obtenidos experimentalmente con aquellos que son de esperarse teóricamente en virtud de las hipótesis

\section{Procedimiento}

En esta investigación se ha optado por dotar al cuestionario utilizado con la mayor rigurosidad, fiabilidad y validez posibles mediante un método de expertos, el método Delphi, utilizando como fuente de información un grupo de personas a las que se supone un conocimiento elevado de la materia que se va a tratar.

En un Delphi existen circulaciones o fases, durante las cuales existe un proyecto inicial de cuestionario que se va redactando y perfilando a través de nuevas sugerencias, modificaciones o supresiones de todos los componentes del panel de expertos, hasta llegar a un cuestionario definitivo, aceptado por todos. Para una mejor apreciación de manera visual, exponemos en la siguiente figura el proceso habitual en el método Delphi:

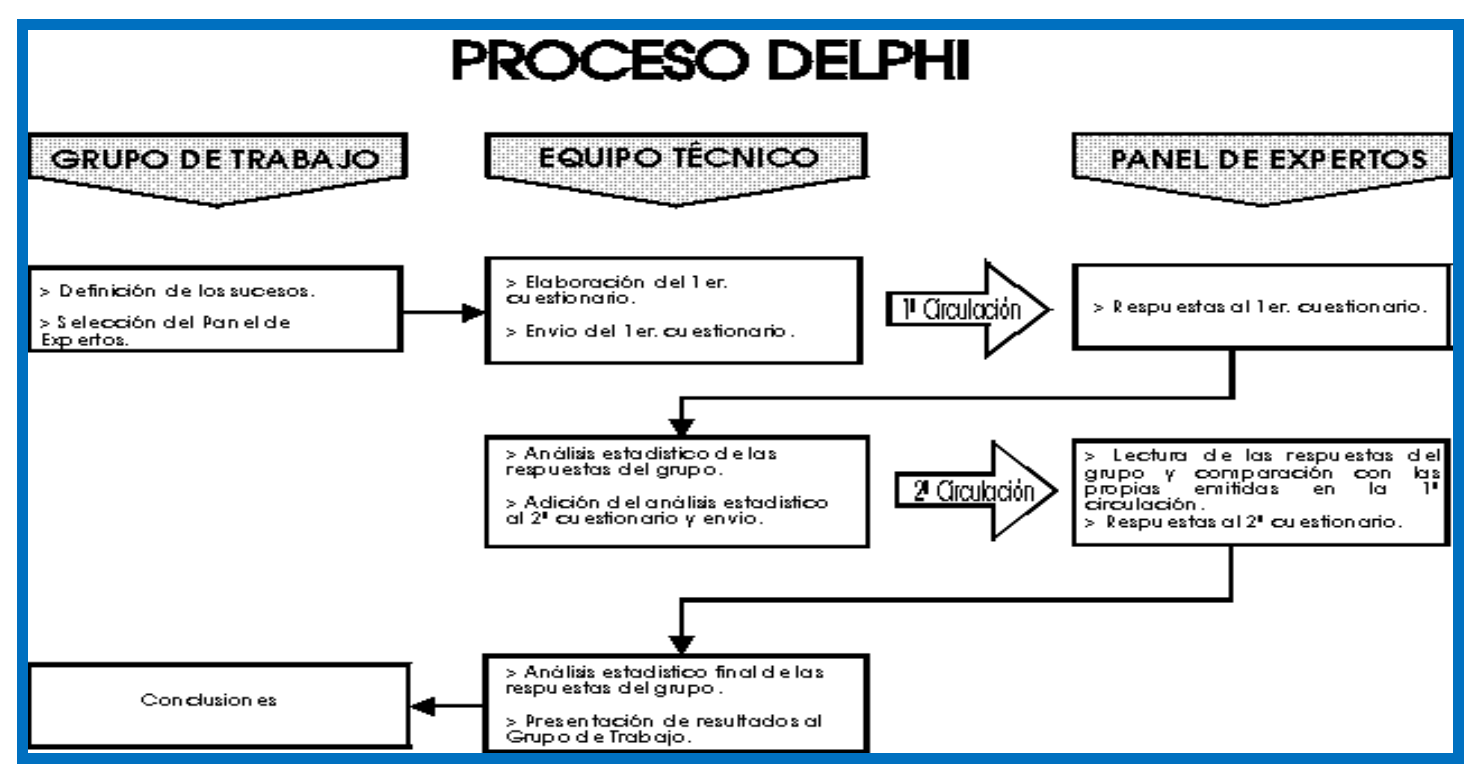

Figura 1. Método Delphi. Fuente de consulta: Grupo Delphos (http://www.grupodelphos.org/) 
Los expertos colaboradores en nuestra investigación para confeccionar el cuestionario utilizado en la recogida de datos han sido (por orden alfabético): Jordi Adell, Manuel Área Moreira, Antonio Bartolomé Pina, $\mathrm{M}^{\mathrm{a}}$ Teresa Bestene, Pablo Bongiovanni, Linda J. Castañeda, Ricardo Fernández Muñoz, Ma Luisa Gómez, Claudia Larrotta, Ana Lorenzo Pedraza, Pere Marquès, Daniela Melaré Vieira Barros, Toni Perulles i Rull, $\mathrm{M}^{\mathrm{a}}$ Mar Rodríguez Albertus, Hernán Soto Núñez.

Hay que reseñar que la práctica totalidad de expertos implicados estaba de acuerdo en que cualquiera de estos cuestionarios sólo es útil cuando se aplica en un contexto determinado, sin la posibilidad de generalización total sin tener en cuenta las características específicas de cada momento y lugar donde se investigue. Por ello, siempre será recomendable que los cuestionarios vayan acompañados de una ficha rellena por el profesor correspondiente donde quedarán reflejados los datos más importantes respecto a la utilización del edublog estudiado: asignatura, curso, especialidad, centro, año escolar, horas semanales de la asignatura, número total de alumnos matriculados, localidad, provincia, país, objetivos, metodología, evaluación, observaciones.

\section{Diseño y Análisis de datos}

La Informática, y más concretamente los paquetes de programas estadísticos consistentes en programas complejos que funcionan bajo el control del usuario, cumplen una inestimable función de ayuda para los investigadores en la fase de análisis de datos. Los paquetes más aplicados en las Ciencias Sociales son el SPSS (Statistical Package for the Social Sciencies) y el BMDP (Biomedical Computers Programs). Todos los datos y gráficos extraídos en este trabajo de investigación se han realizado mediante el SPSS (versión 15.0).

Para realizar el análisis descriptivo de datos se han utilizado tablas de frecuencia con gráficos (frecuencia absoluta, porcentaje, porcentaje válido, porcentaje acumulado) medidas de tendencia central (media aritmética, mediana, moda, desviación típica, varianza, rango, percentiles) histogramas, variabilidad, asimetría y curtosis.

En cuanto a la estadística, en el estudio de las variables bidimensionales y, en general, también de las multidimensionales, es a menudo bastante interesante investigar la posible existencia de alguna relación de dependencia entre las variables. Para ello, se han llevado a 
cabo diversas pruebas que han ayudado en la investigación del tema que nos ocupa. Por un lado, se establecieron relaciones de contenidos entre sí intentando descubrir la existencia o no de dicha dependencia. Posteriormente, se averiguaron los coeficientes de correlación mediante modelos de regresión tanto paramétricos como no paramétricos (coeficiente de correlación de Pearson, coeficiente de correlación lineal rho de Spearman, coeficiente de correlación tau de kendall, prueba de Chi-cuadrado), pudiendo utilizarse los resultados obtenidos para las posibles predicciones.

\section{Resultados}

Intentando simplificar y concretar todo este trabajo, expondremos a continuación algunas conclusiones que actúan como punto de unión y enlace entre el problema de investigación tratado, los objetivos perseguidos y los resultados obtenidos a través de las respuestas ofrecidas por la muestra productora de datos a las preguntas de investigación.

Teniendo en cuenta las opiniones y valoraciones del alumnado y profesorado colaboradores en el estudio, podemos enumerar una serie de afirmaciones en cuanto a la contribución de los edublogs en el ámbito educativo: la utilización de un edublog en el aula estimula el seguimiento de la asignatura, favorece la solución de dudas en cuanto a la asignatura, estimula la búsqueda de información nueva sobre el tema, facilita la expresión de ideas y opiniones sobre temas de actualidad relacionados con la asignatura, facilita información importante de otras actividades relacionadas con la asignatura, fomenta la participación de los estudiantes en la asignatura, promueve el pensamiento crítico, favorece el pensamiento creativo, facilita el contacto con el profesor estableciendo una relación fluida y regular, potencia la colaboración con el profesor, facilita la comunicación entre los estudiantes, fomenta las relaciones personales con los compañeros, favorece la interacción y el trabajo colaborativo entre los estudiantes, promueve que el estudiante se sienta parte del grupo, estimula nuevas formas de relación, posibilita la relación con otras personas ajenas a nuestro grupo-clase, aumenta el interés por la asignatura, fomenta la valoración positiva de la asignatura, estimula a realizar las actividades propias de la asignatura, anima a los compañeros a participar, conecta los contenidos de la asignatura con la actualidad, promueve la profundización en el tema, favorece una mayor implicación de los alumnos en las tareas de aprendizaje, fomenta la participación en otras actividades o eventos ajenos a la asignatura, favorece la adquisición de contenidos de 
la asignatura, facilita la puesta en práctica de contenidos de la asignatura, facilita la integración y aprendizaje de contenidos transversales, ayuda al desarrollo de la habilidad lectora, ayuda al desarrollo de la habilidad escritora, ayuda al desarrollo de la expresión oral, favorece la creación de Comunidades de estudiantes, favorece la construcción de nuevos conocimientos (nuevo pensamiento).

Dada la imposibilidad de incorporar en estas páginas la cantidad ingente de tablas y gráficos existentes, nos limitaremos a mostrar algunos que, dado su carácter generalizador, servirán de muestra y prueba de los resultados obtenidos.

A continuación, en las páginas siguientes, se pueden ver las tres figuras que resumen los resultados obtenidos en este trabajo de investigación:

\section{Valoración global del edublog como herramienta educativa.}

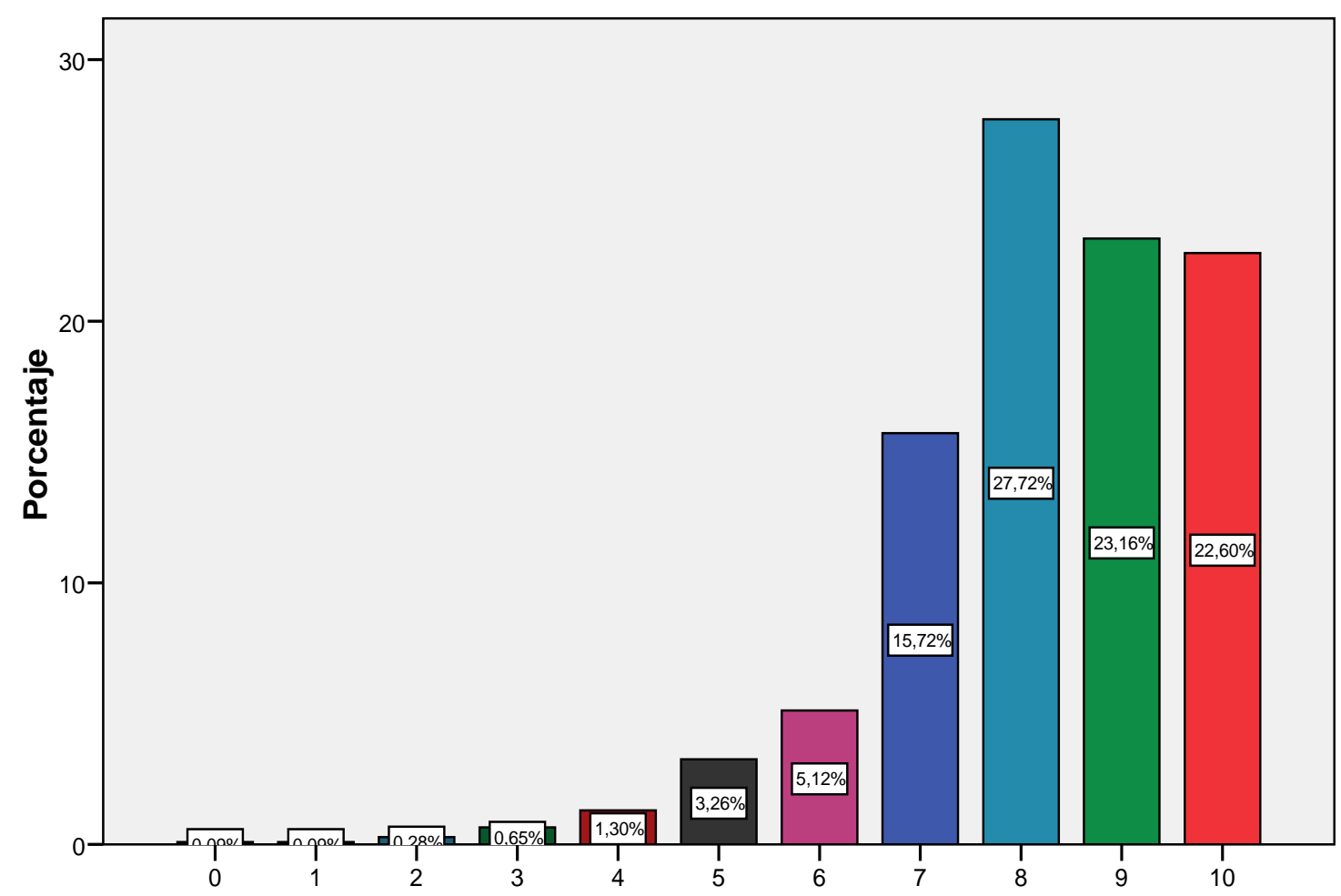

Figura 2. Valoración global del edublog como herramienta educativa.

Especificando que la escala de puntuación se estableció entre 0 (valoración negativa mínima) hasta el 10 (valoración positiva máxima), en este primer gráfico podemos comprobar 
la notable valoración que la muestra otorga a los edublogs como herramientas educativas, acumulándose la inmensa mayoría de respuestas entre el 7 y 10.

Utilizando la misma escala de puntuación entre 0 y 10, la valoración global por parte de la muestra en cuanto al uso que han hecho los profesores de los edublogs es aún más destacable que la anterior, como podemos observar en este segundo gráfico, donde el mayor número de respuestas se ha establecido en la valoración máxima (10). También se puede destacar el ínfimo número de valoraciones por debajo del 7.

Valoración global del uso que ha hecho del edublog el profesor.

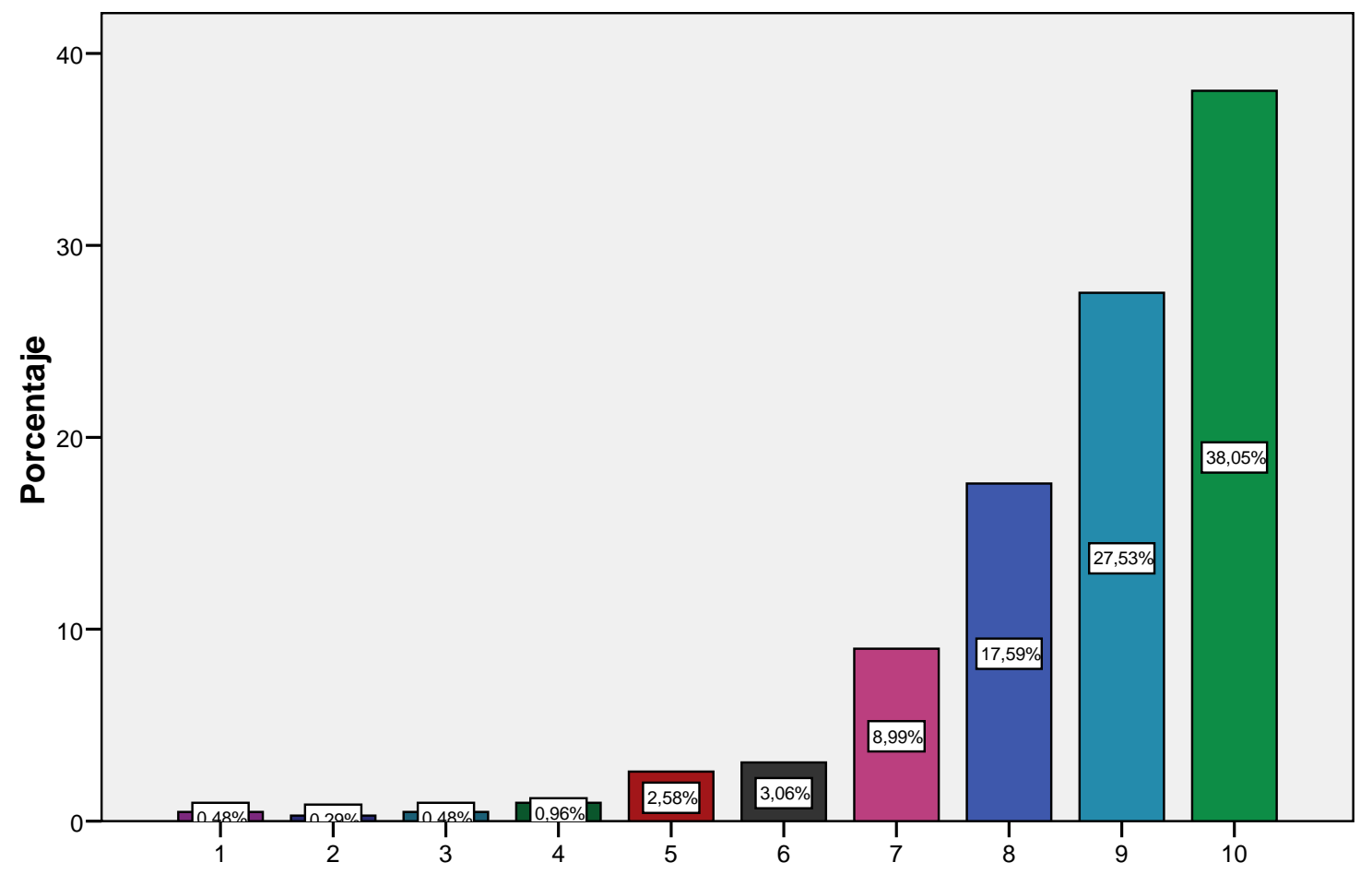

Figura 3. Valoración global del uso que ha hecho del edublog el profesor.

Por último, en el siguiente y último gráfico que podemos observar a continuación, en la siguiente página, comprobamos la valoración que se hace más concretamente de nuestro objeto de estudio: la contribución de los edublogs como estrategia didáctica favorecedora de la adquisición de contenidos. De igual manera que en los gráficos anteriores, la valoración por parte de la muestra es inequívocamente favorable, constatando un elevado número de colaboradores totalmente y medianamente de acuerdo. 


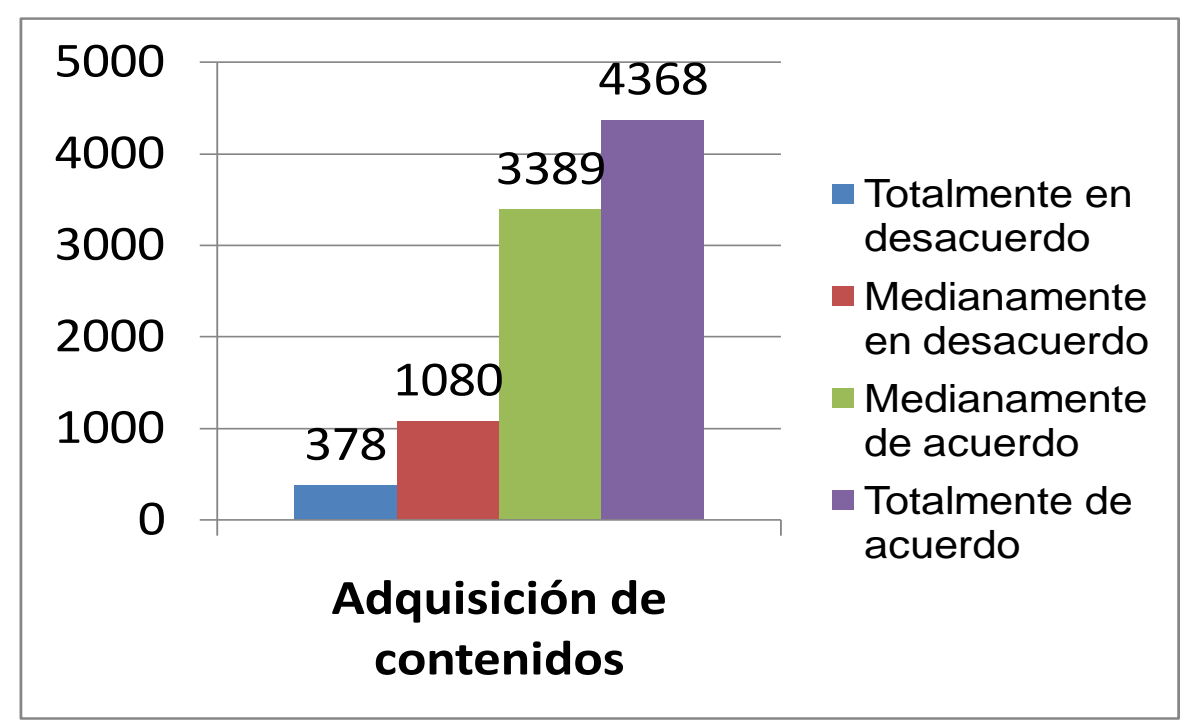

Figura 4. Valoración global del edublog como estrategia didáctica facilitadora de adquisición de contenidos.

\section{Discusión y Conclusiones}

Un aspecto a mejorar, deseable en cualquier investigación pero ausente en ésta, es la utilización de varios grupos (o varios test al mismo grupo) en vez de uno solo. Los resultados hubieran sido más contundentes, más visibles y contrastables si se hubiera dispuesto de una de las dos opciones siguientes:

Haber dispuesto de un grupo experimental y otro de control donde la única diferencia fuera que en el primero de ellos se hubiera trabajado utilizando un edublog de aula y en el segundo sin él. Y al final del curso se pasarían unos cuestionarios idénticos para los dos grupos sin hacer mención a la utilización del edublog donde simplemente tendrían que puntuar diversos ítems que valoraran los cuatro grandes objetivos tratados (motivación, relaciones personales, dinamización del proceso de Enseñanza-Aprendizaje y adquisición de contenidos) o como mínimo las dos hipótesis planteadas en el título del trabajo: la motivación y las relaciones personales. 
Del mismo modo, también se podría haber realizado un pre-test y un post-test con el mismo grupo, antes y después de haber utilizado el edublog de aula para averiguar los posibles cambios en los alumnos en cuanto a los aspectos estudiados.

De esta manera, se podrían comparar los datos obtenidos por ambos grupos (o por el mismo grupo pero en los distintos cuestionarios) y comprobar si verdaderamente la utilización del edublog es la que determina los resultados más favorables de uno u otro grupo o si, por el contrario, los resultados son similares en ambos casos.

Es evidente que en ambos casos se aísla la variable 'utilización del edublog' haciendo que los resultados obtenidos sean determinados exclusivamente por dicha variable y, por tanto, desechando otras difíciles de valorar que habrían podido influir en los mismos, como por ejemplo podrían ser la personalidad de los distintos profesores, los distintos enfoques que dan a sus asignaturas, la peculiar forma de cada uno de llevar sus clases, etc.

Aun así, todo lo expuesto en este estudio nos lleva a deducir que la utilización del edublog, independientemente de otras muchas variables (como pueden ser el sexo, la obligatoriedad de los estudios, tipo de localidad, nivel de utilización, etc.), sí resulta eficaz como estrategia didáctica facilitadora de adquisición de contenidos.

Y para concretar más, se puede exponer la clasificación gradual de mayor a menor acuerdo teniendo en cuenta el valor otorgado por la muestra a los diferentes campos de estudio dentro de la dinamización del proceso. De esta manera, algunas conclusiones que reflejan la consecución de los objetivos propuestos son: la utilización de un edublog en el aula favorece la adquisición de contenidos de la asignatura, facilita la puesta en práctica de contenidos de la asignatura, facilita la integración y aprendizaje de contenidos transversales, ayuda al desarrollo de la habilidad lectora, ayuda al desarrollo de la habilidad escritora, favorece la construcción de nuevos conocimientos (nuevo pensamiento), favorece la creación de Comunidades de estudiantes y ayuda al desarrollo de la expresión oral. 


\section{Referencias}

Cacheiro, M. L. (2002). Multimedia: un nuevo lenguaje. En Actas del Congreso Tecnologías de la Información y la Comunicación para el Aprendizaje (pp. 495-507). Madrid: UNED.

Clandinin, J. y Conelly, F. M. (1986). The reflective practitioner and practitioners narrative units. Canadian journal of education. 11(2), 184-198.

Dilmac, B. (2009). Un análisis del profesorado: su tendencia general a la dilación, percepción de eficacia profesional / auto-eficacia y altruismo. Electronic Journal of Research in Educational Psychology, 19, 7(3), 1323-1338.

García, A. (2008). Edublogs: Un enfoque globalizador de las competencias básicas en el currículo de lengua extranjera. Pulso: revista de Educación, 31, 117-137.

García, M. M. y Romero, I. M. (2009). Influencia de las nuevas tecnologías en la evolución del aprendizaje y las actitudes matemáticas de estudiantes de secundaria. Electronic Journal of Research in Educational Psychology, 17, 7(1), 369-396.

Gértrudix, F. y Gértrudix, M. (2007). Investigaciones entorno a las tic en educación: una panorámica actualizada. Docencia e Investigación, segunda época, 17, 119-146.

Gómez, M. (2004 Septiembre 22). Colaboración en red con soporte tecnológico (TIC) en la resolución de problemas de Matemáticas. Quaderns Digitals, Monográfico: Educación a Distancia, Artículo 5. Extraído el 1 abril, 2010, de http://www.quadernsdigitals.net/index.php?accionMenu=hemeroteca.VisualizaArticul oIU.visualiza\&articulo_id $=7748$

Michel, B. (2009 Junio 6). Edublog propuesta interactiva de la reforma educativa en Educación Primaria. Blogger. Extraído el 1 abril, 2010, de http://edublog7.blogspot.com/

Sánchez, J. (1999). Nuevas tecnologías (NNTT) y enseñanza. Quaderns Digitals, 20, Artículo 9. Extraído el 1 abril, 2010, de http://www.quadernsdigitals.net/index.php?accionMenu=hemeroteca.VisualizaArticul oIU.visualiza\&articulo_id=134

Uribe, L.F. (2009). El impacto de los edublogs y la inteligencia colectiva en los procesos educativos. Ponencia presentada en el IV Congreso de la Cibersociedad 2009. Cibersociedad. Extraído el 1 abril, 2010, de http://www.cibersociedad.net/congres2009/es/coms/el-impacto-de-los-edublogs-y-lainteligencia-colectiva-en-los-procesos-educativos/520/ 


\section{Anexo I: Cuestionario.}

\section{CUESTIONARIO SOBRE LA UTILIZACIÓN DE UN BLOG EN LA ASIGNATURA} (ENSEÑANZA OBLIGATORIA)

FICHA A RELLENAR POR EL PROFESORADO (UNA POR CADA CURSO Y/O ASIGNATURA)

ASIGNATURA:

CURSO:

ESPECIALIDAD:

CENTRO:

AÑO ESCOLAR:

HORAS SEMANALES DE LA ASIGNATURA:

No TOTAL DE ALUMNOS/AS MATRICULADOS/AS EN LA ASIGNATURA:

LOCALIDAD, PROVINCIA, PAÍS:

DESCRIPCIÓN DE LA ACTIVIDAD CON EL BLOG (METODOLOGÍA):

OBJETIVOS PERSEGUIDOS CON LA CREACIÓN Y UTILIZACIÓN DEL BLOG:

VALORACIÓN DE LA ACTIVIDAD (EVALUACIÓN):

OBSERVACIONES: 


\section{CUESTIONARIO SOBRE LA UTILIZACIÓN DE UN BLOG EN LA ASIGNATURA} (ENSEÑANZA OBLIGATORIA)

FICHA A RELLENAR POR EL ALUMNADO

(Cliquear con el ratón en las casillas correspondientes)

EDAD: $\square$ Menos de 18 años.

35 - 45 años. $\square 25$ - 35 años.

Más de 55 años.

SEXO: $\square$ Hombre. $\square$ Mujer.

ESTUDIOS QUE REALIZAS: $\square$ Bachillerato. $\quad \square$ Ciclo Formativo Grado Medio.

$\square$ Universidad. $\quad \square$ Ciclo Formativo Grado Superior.

NE.

\section{¿CÓMO CONSIDERAS QUE ES TU NIVEL DE UTILIZACIÓN DEL ORDENADOR?:}

No sé ni encenderlo.

Sólo lo uso en caso de necesidad,utilizando únicamente los programas básicos.

Lo uso bastante y me defiendo con soltura, aunque estoy limitado a sólo algunos programas.

$\square$ El ordenador no tiene secretos para mí, lo uso casi todo el tiempo y me defiendo muy bien.

\section{¿DESDE DÓNDE SUELES ACCEDER A INTERNET Y AL BLOG?:}

$\square$ Casa.

$\square$ Centro Educativo.

Cybercafé.

Amigos.

Otros.

¿Tienes ordenador en casa?

$\square$ Sí.

$\square$ No.

Si la anterior respuesta ha sido afirmativa. ¿Tienes conexión a Internet?...

Sí.

$\square$ No.

Si la anterior respuesta ha sido afirmativa. ¿Tienes línea de alta velocidad?

Sí.

$\square$ No.

¿Tienes conocimiento de la existencia de un Blog en la asignatura?.

DSí.

$\square$ No.

¿Tienes tu propio Blog?.

$\square$ Sí.

$\square$ No.

¿Has visitado el Blog de la asignatura?.......... $\square$ Muchas veces. $\square$ Bastantes. $\square$ Pocas. $\square$ Ninguna.
¿Has leído entradas?........................................ $\square$ Muchas veces. $\square$ Bastantes. $\square$ Pocas. $\square$ Ninguna.
¿Has leído comentarios?................................. $\square$ Muchas veces. $\square$ Bastantes. $\square$ Pocas. $\square$ Ninguna.
¿Has escrito entradas?.................................... $\square$ Muchas veces. $\square$ Bastantes. $\square$ Pocas. $\square$ Ninguna.
¿Has escrito comentarios?.............................. $\square$ Muchas veces. $\square$ Bastantes. $\square$ Pocas. $\square$ Ninguna.
¿Has insertado imágenes en el Blog?............ $\square$ Muchas veces. $\square$ Bastantes. $\square$ Pocas. $\square$ Ninguna.




\begin{tabular}{|c|c|c|c|c|}
\hline DINAMIZACIÓN DEL PROCESO ENSEÑANZA-APRENDIZAJE & 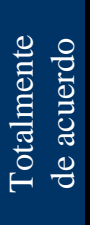 & 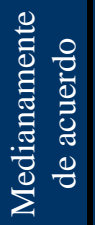 & 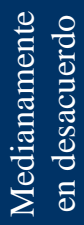 & 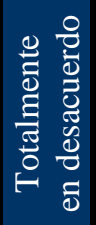 \\
\hline \multicolumn{5}{|l|}{ Estimula el seguimiento de la asignatura. } \\
\hline \multicolumn{5}{|l|}{ Favorece la solución de dudas en cuanto a la asignatura. } \\
\hline \multicolumn{5}{|l|}{ Estimula la búsqueda de información nueva sobre el tema. } \\
\hline \multicolumn{5}{|l|}{$\begin{array}{l}\text { Facilita la expresión de ideas y opiniones sobre temas de actualidad relacionados } \\
\text { con la asignatura. }\end{array}$} \\
\hline \multicolumn{5}{|l|}{$\begin{array}{l}\text { Facilita información importante de otras actividades relacionadas con la asigna- } \\
\text { tura. }\end{array}$} \\
\hline \multicolumn{5}{|l|}{ Fomenta la participación de los estudiantes en la asignatura. } \\
\hline \multicolumn{5}{|l|}{ Promueve el pensamiento crítico. } \\
\hline \multicolumn{5}{|l|}{ Favorece el pensamiento creativo. } \\
\hline (Si quieres señalar algo que no se encuentre en el cuestionario, puedes utilizar estas 2 últimas líne & & & & \\
\hline & & & & \\
\hline
\end{tabular}

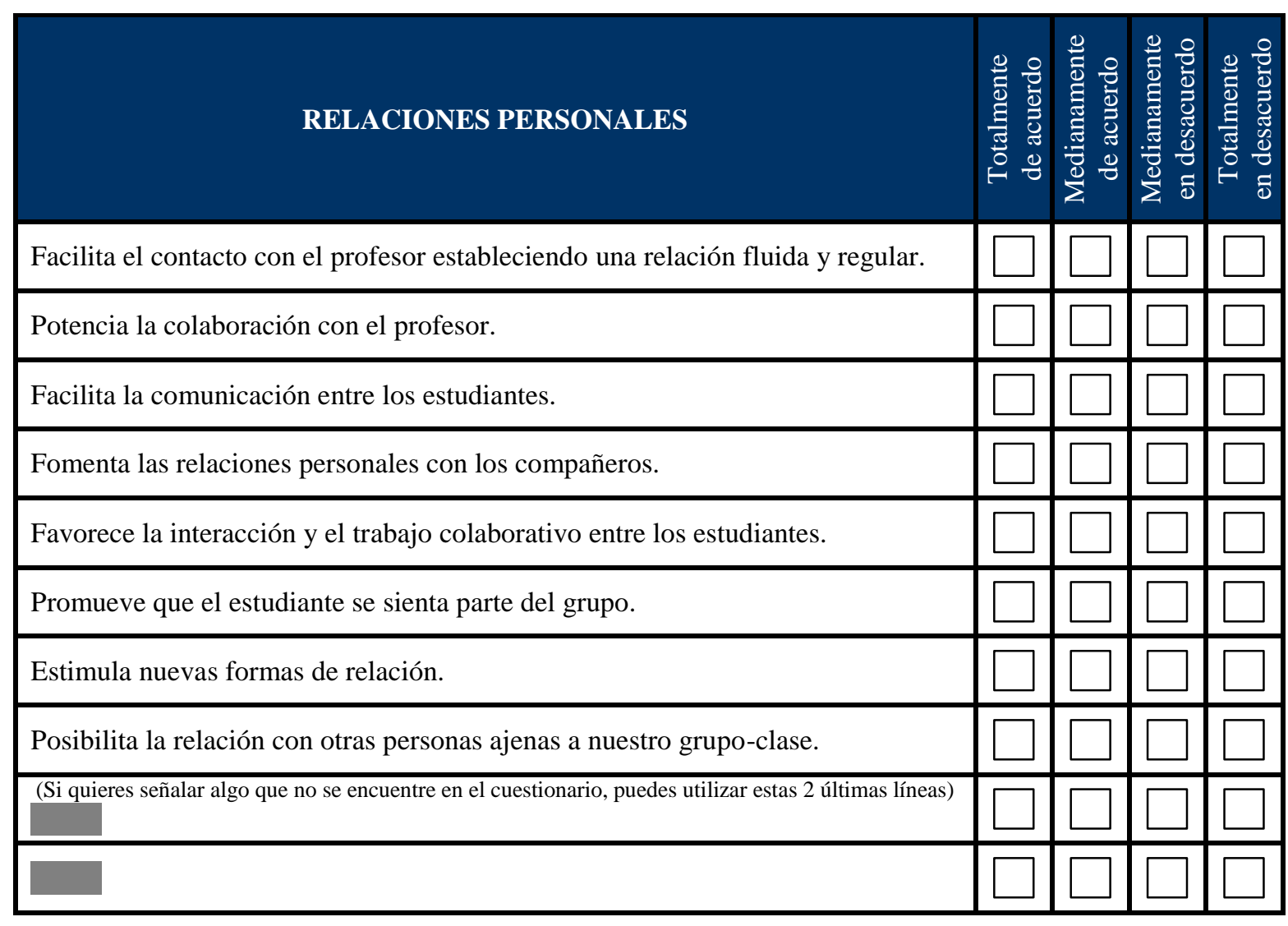




\begin{tabular}{|c|c|c|c|c|}
\hline MOTIVACIÓN & 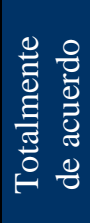 & 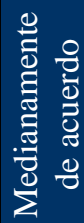 & 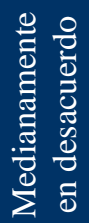 & 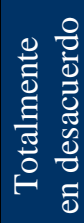 \\
\hline \multicolumn{5}{|l|}{ Aumenta el interés por la asignatura. } \\
\hline \multicolumn{5}{|l|}{ Fomenta la valoración positiva de la asignatura. } \\
\hline \multicolumn{5}{|c|}{ Estimula a realizar las actividades propias de la asignatura. } \\
\hline \multicolumn{5}{|l|}{ Anima a los compañeros a participar. } \\
\hline \multicolumn{5}{|c|}{ Conecta los contenidos de la asignatura con la actualidad. } \\
\hline \multicolumn{5}{|l|}{ Promueve la profundización en el tema. } \\
\hline \multicolumn{5}{|c|}{ Favorece una mayor implicación de los alumnos en las tareas de aprendizaje. } \\
\hline \multicolumn{5}{|c|}{ Fomenta la participación en otras actividades o eventos ajenos a la asignatura. } \\
\hline (Si quieres señalar algo que no se encuentre en el cuestionaric & & & & \\
\hline & & & & \\
\hline
\end{tabular}

\begin{tabular}{|c|c|c|c|c|}
\hline ADQUISICIÓN DE CONTENIDOS & 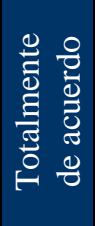 & 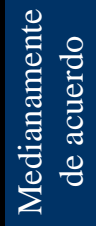 & 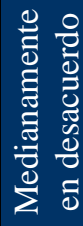 & 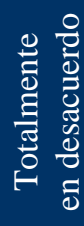 \\
\hline \multicolumn{5}{|l|}{ Favorece la adquisición de contenidos de la asignatura. } \\
\hline \multicolumn{5}{|l|}{ Facilita la puesta en práctica de contenidos de la asignatura. } \\
\hline \multicolumn{5}{|l|}{ Facilita la integración y aprendizaje de contenidos transversales. } \\
\hline \multicolumn{5}{|l|}{ Ayuda al desarrollo de la habilidad lectora. } \\
\hline \multicolumn{5}{|l|}{ Ayuda al desarrollo de la habilidad escritora. } \\
\hline \multicolumn{5}{|l|}{ Ayuda al desarrollo de la expresión oral. } \\
\hline \multicolumn{5}{|l|}{ Favorece la creación de Comunidades de estudiantes. } \\
\hline \multicolumn{5}{|c|}{ Favorece la construcción de nuevos conocimientos (nuevo pensamiento). } \\
\hline (Si quieres señalar algo que no se encuentre en el cuestionario, puedes utiliz & & & & \\
\hline & & & & \\
\hline
\end{tabular}

VALORACIÓN GLOBAL

DEL BLOG COMO HERRAMIENTA
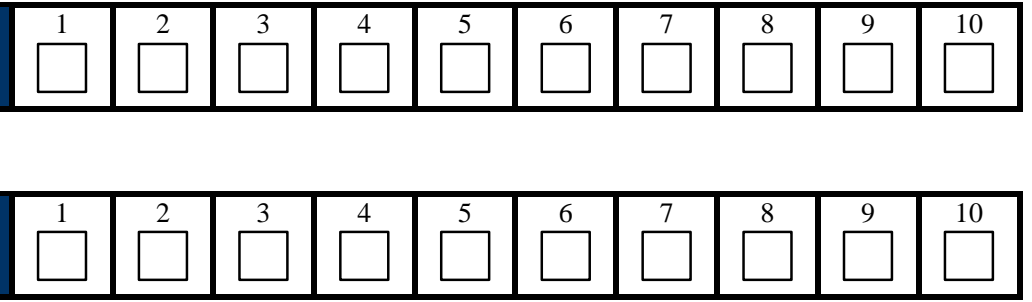

$-352-$

Electronic Journal of Research in Educational Psychology, 9(1), 331-356. ISSN: 1696-2095. 2011, no. 23 


\section{Anexo II: Correlaciones.}

Edad - Adquisición de contenidos.

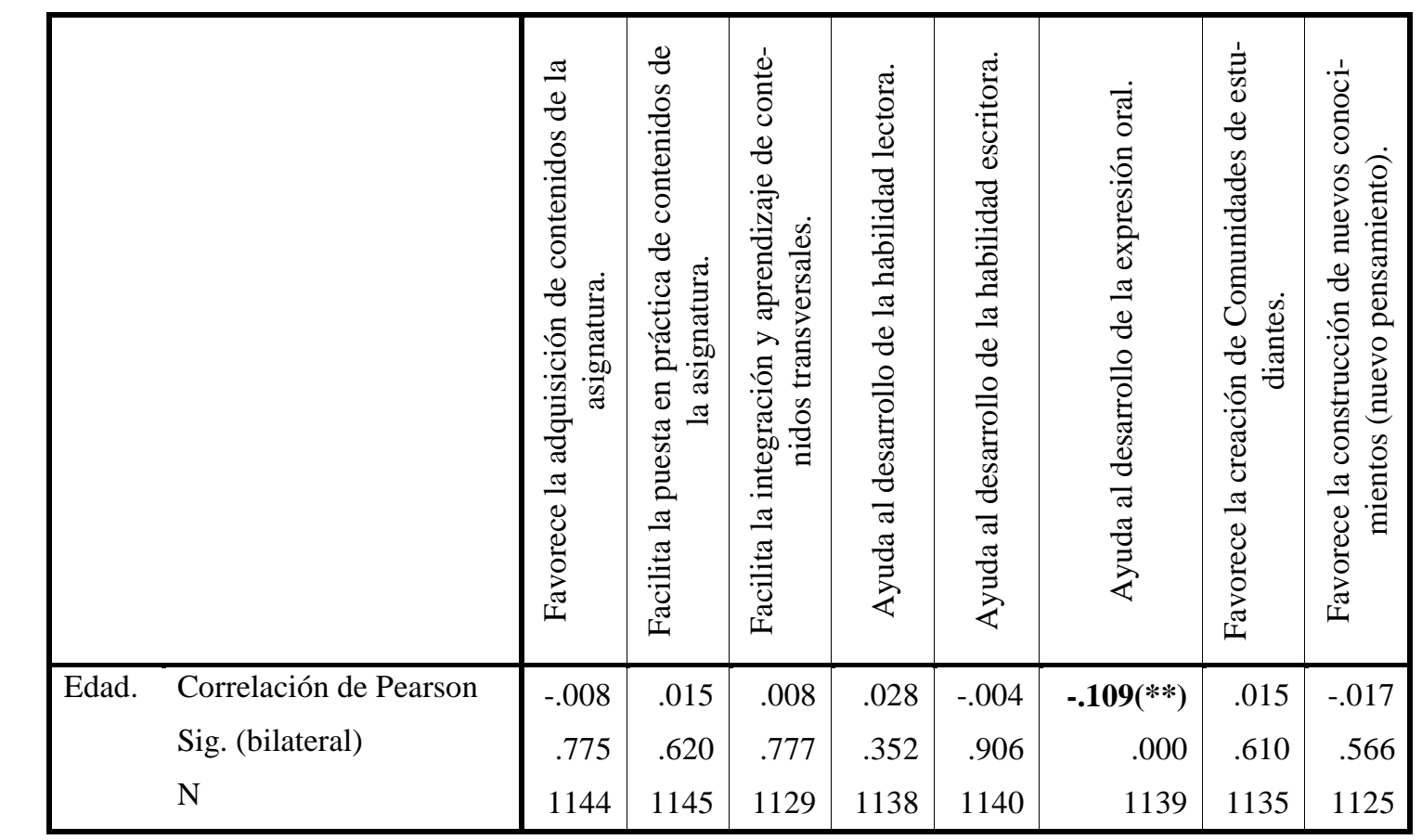

** La correlación es significativa al nivel .01 (bilateral).

Correlaciones no paramétricas: Edad - Adquisición de contenidos.

\begin{tabular}{|c|c|c|c|c|c|c|c|c|c|c|}
\hline & & & 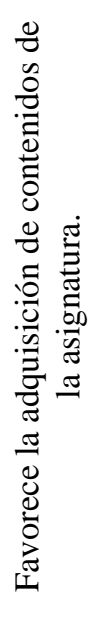 & 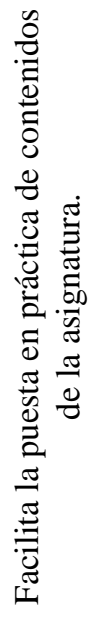 & 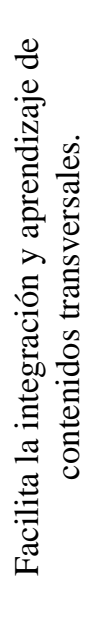 & 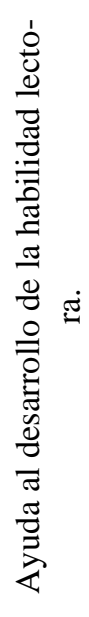 & 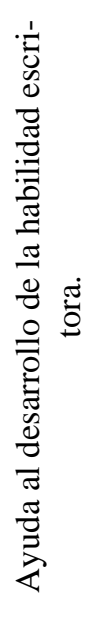 & 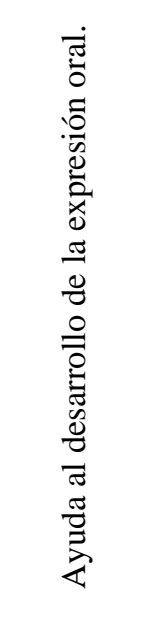 & 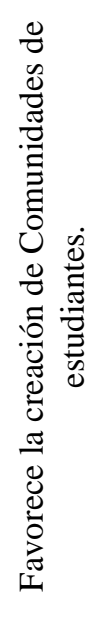 & 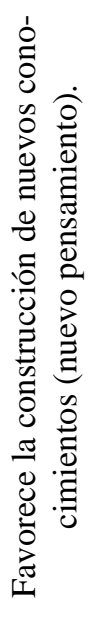 \\
\hline \multirow[t]{3}{*}{$\begin{array}{l}\text { Tau_b de Ken } \\
\text { dall }\end{array}$} & Edad. & $\begin{array}{l}\text { Coefic. de } \\
\text { correlación }\end{array}$ & -.013 & .007 & $.00 \overline{3}$ & .013 & .018 & $-.097(* *)$ & .017 & -.016 \\
\hline & & Sig. (bil.) & .619 & .789 & .909 & .605 & .471 & .000 & .488 & .528 \\
\hline & & $\mathrm{N}$ & 1144 & 1145 & 1129 & 1138 & 1140 & 1139 & 1135 & 1125 \\
\hline \multirow[t]{3}{*}{$\begin{array}{l}\text { Rho de Spear- } \\
\text { man }\end{array}$} & Edad. & $\begin{array}{l}\text { Coef. de } \\
\text { correlación }\end{array}$ & -.015 & .008 & .004 & .014 & $.022^{-}$ & $-.120(* *)$ & .020 & -.019 \\
\hline & & Sig. (bil.) & .615 & .793 & .883 & .646 & .463 & .000 & .504 & .524 \\
\hline & & $\mathrm{N}$ & 1144 & 1145 & 1129 & 1138 & 1140 & 1139 & 1135 & 1125 \\
\hline
\end{tabular}

** La correlación es significativa al nivel .01 (bilateral). 


\section{Anexo III: Chi- Cuadrado.}

Pruebas de chi-cuadrado: Favorece la adquisición de contenidos de la asignatura.

\begin{tabular}{|c|c|c|c|}
\hline $\begin{array}{c}\text { Favorece la adquisición de contenidos de la asignatura / Tipo } \\
\text { de localidad: rural o urbana. }\end{array}$ & Valor & gl & Sig. \\
\hline Chi-cuadrado de Pearson & 3,355 & 3 & .340 \\
\hline
\end{tabular}

\begin{tabular}{|c|c|c|c|}
\hline $\begin{array}{c}\text { Favorece la adquisición de contenidos de la asignatura / } \\
\text { Obligatoriedad de los estudios. }\end{array}$ & Valor & gl & Sig. \\
\hline Chi-cuadrado de Pearson & 953 & 3 & .813 \\
\hline
\end{tabular}

\begin{tabular}{|c|c|c|c|}
\hline $\begin{array}{c}\text { Favorece la adquisición de contenidos de la asignatura / } \\
\text { Nivel de utilización del ordenador. }\end{array}$ & Valor & gl & Sig. \\
\hline Chi-cuadrado de Pearson & 5,347 & 9 & .803 \\
\hline
\end{tabular}

\begin{tabular}{|c|c|c|c|}
\hline $\begin{array}{c}\text { Favorece la adquisición de contenidos de la asignatura / } \\
\text { Desde dónde suele acceder al edublog. }\end{array}$ & Valor & gl & Sig. \\
\hline Chi-cuadrado de Pearson & 12,999 & 15 & .602 \\
\hline
\end{tabular}

\begin{tabular}{|c|c|c|c|}
\hline $\begin{array}{c}\text { Favorece la adquisición de contenidos de la asignatura / } \\
\text { ¿Tiene ordenador en casa? }\end{array}$ & Valor & gl \\
\hline Chi-cuadrado de Pearson & 6,586 & 3 & .086 \\
\hline
\end{tabular}

\begin{tabular}{|c|c|c|c|}
\hline $\begin{array}{c}\text { Favorece la adquisición de contenidos de la asignatura / } \\
\text { ¿Tiene conexión a Internet? }\end{array}$ & Valor & gl \\
\hline Chi-cuadrado de Pearson & 7,602 & 3 & .055 \\
\hline
\end{tabular}

\begin{tabular}{|c|c|c|c|}
\hline $\begin{array}{c}\text { Favorece la adquisición de contenidos de la asignatura / } \\
\text { ¿Tiene línea de alta velocidad? }\end{array}$ & Valor & gl & Sig. \\
\hline Chi-cuadrado de Pearson & 7,027 & 3 & .071 \\
\hline
\end{tabular}




\begin{tabular}{|c|c|c|c|}
\hline $\begin{array}{c}\text { Favorece la adquisición de contenidos de la asignatura / } \\
\text { ¿Tiene su propio blog? }\end{array}$ & Valor & gl & Sig. \\
\hline Chi-cuadrado de Pearson & 4,598 & 3 & .204 \\
\hline
\end{tabular}

\begin{tabular}{|c|c|c|c|}
\hline $\begin{array}{c}\text { Favorece la adquisición de contenidos de la asignatura / ¿Ha } \\
\text { escrito comentarios en el edublog? }\end{array}$ & Valor & gl & Sig. \\
\hline Chi-cuadrado de Pearson & 14,774 & 9 & .097 \\
\hline
\end{tabular}

\begin{tabular}{|c|c|c|c|}
\hline $\begin{array}{c}\text { Favorece la adquisición de contenidos de la asignatura / ¿Ha } \\
\text { insertado imágenes en el edublog? }\end{array}$ & Valor & gl & Sig. \\
\hline Chi-cuadrado de Pearson & 15,217 & 9 & .085 \\
\hline
\end{tabular}


José Francisco Durán Medina

[Página en blanco por razones de paginación] 